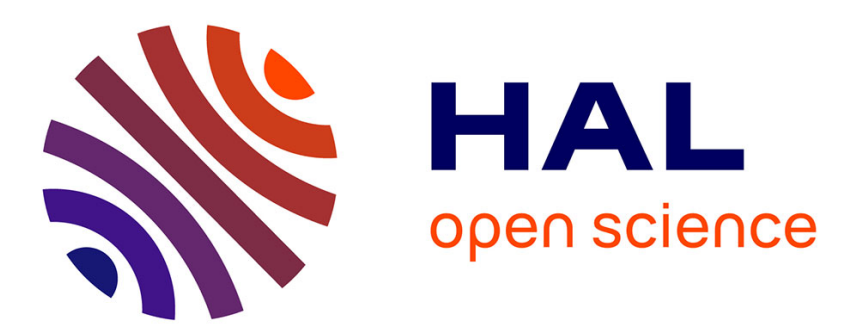

\title{
Competing Four-Wave Mixing Processes in Dispersion Oscillating Telecom Fiber
}

Christophe Finot, Julien Fatome, Alexej A. Sysoliatin, Alexey Fedorovich Kosolapov, Stefan Wabnitz

\section{- To cite this version:}

Christophe Finot, Julien Fatome, Alexej A. Sysoliatin, Alexey Fedorovich Kosolapov, Stefan Wabnitz. Competing Four-Wave Mixing Processes in Dispersion Oscillating Telecom Fiber. Optics Letters, 2013, 38 (24), pp.5361-5364. 10.1364/OL.38.005361 . hal-00871601

\section{HAL Id: hal-00871601 \\ https://hal.science/hal-00871601}

Submitted on 14 Oct 2013

HAL is a multi-disciplinary open access archive for the deposit and dissemination of scientific research documents, whether they are published or not. The documents may come from teaching and research institutions in France or abroad, or from public or private research centers.
L'archive ouverte pluridisciplinaire HAL, est destinée au dépôt et à la diffusion de documents scientifiques de niveau recherche, publiés ou non, émanant des établissements d'enseignement et de recherche français ou étrangers, des laboratoires publics ou privés. 


\title{
Competing Four-Wave Mixing Processes in Dispersion Oscillating Telecom Fiber
}

\author{
Christophe Finot, ${ }^{1,}$ Julien Fatome, ${ }^{1}$ Alexej Sysoliatin, ${ }^{2}$ A. Kosolapov, ${ }^{2}$ and Stefan Wabnitz ${ }^{3}$ \\ ${ }^{1}$ Laboratoire Interdisciplinaire Carnot de Bourgogne, UMR 6303, 9 av. Alain Savary, 21078 Dijon, France \\ ${ }^{2}$ Fiber Optics Research Center, 11933 Moscow, Russia \\ ${ }^{3}$ Dipartimento di Ingegneria dell'Informazione, Università degli Studi di Brescia, via Branze 38, 25123, Brescia, Italy \\ *Corresponding author: christophe.finot@u-bourgogne.fr \\ Received Month X, XXXX; revised Month X, XXXX; accepted Month X, \\ XXXX; posted Month X, XXXX (Doc. ID XXXXX); published Month X, XXXX
}

\begin{abstract}
We experimentally study the dynamics of the generation of multiple sidebands by means of a quasi-phase-matched fourwave mixing process occurring in a dispersion oscillating, highly nonlinear optical fiber. The fiber under test is pumped by a ns microchip laser operating in the normal average group-velocity dispersion regime and in the telecom $\mathrm{C}$ band. We reveal that the growth of higher order sidebands is strongly influenced by the competition with cascade four-wave mixing between the pump and the first order quasi-phase matched sidebands. The properties of these competing four wave mixing processes are substantially affected when a partially coherent pump source is used, leading to a drastic reduction of the average power needed for sideband generation.

OCIS Codes: (060.4370) Nonlinear optics, fibers, (190.4380) Nonlinear optics, four-wave mixing, (190.4410) Nonlinear optics, parametric process
\end{abstract}

The generation of a set of new optical frequencies from a pump laser has a variety of applications, ranging from optical metrology to spectroscopy and wavelength division multiplexing. Nonlinear methods to generate a regular comb of new frequency components are based on the multiple four-wave mixing (FWM) process occurring in highly nonlinear waveguides [1]. The mechanism for the generation of frequency sidebands from a CW pump is Kerr-induced phase-matching of the FWM interaction or modulation instability (MI), which requires operating in the anomalous dispersion regime. Such restriction can be circumvented with a cavity geometry, where MI [2] and frequency comb generation [3] may also be observed in the normal dispersion regime. Alternatively, one may obtain MI and unequally spaced Kerr frequency comb of sidebands with average normal, periodically varying dispersion, so that the FWM process is quasi-phasematched (QPM) [4-7]. QPM-FWM was recently observed in a dispersion oscillating photonic crystal fiber by using intense $2 \mathrm{~ns}$ pulses from a master optical power oscillator emitting at $1072 \mathrm{~nm}$ [8]. The interplay of QPM-FWM with supercontinuum (SC) generation was also studied in a dispersion oscillating, highly nonlinear fiber with average anomalous dispersion at telecom wavelengths [9]. In this Letter we present the first experimental demonstration of the generation of unequal spacing frequency comb of sidebands based on the QPM-FWM process in average normal, dispersion oscillating fiber (DOF) at telecom wavelengths. Moreover, our experiments reveal that higher-order QPM-FWM sideband generation competes with the cascade or multiple four-wave mixing (MWFM) process which is activated by the mixing of the pump with the first-order QPM-FWM sideband pair.

Pulse propagation in a DOF can be described by the Nonlinear Schrödinger Equation (NLSE)

$$
i \frac{\partial \psi}{\partial z}-\frac{\beta_{2}(z)}{2} \frac{\partial^{2} \psi}{\partial t^{2}}+\gamma|\psi|^{2} \psi=0
$$

Here $z$ and $t$ denote the distance and retarded time (in the frame travelling at the group-velocity) coordinates, $\psi$ is the complex field envelope, $\beta_{2}$ and $\gamma$ are the longitudinally-varying second order group velocity dispersion (GVD) and the constant Kerr nonlinear coefficients, respectively. We have numerically checked that for the fiber parameters and the typical range of powers that will be described in the experimental section, fiber loss and higher order linear and nonlinear effects such as third order dispersion, Raman scattering or selfsteepening have a very limited impact, and therefore can be neglected in a first approximation and for the clarity of the discussion.

In our DOF, the variation of GVD with distance may be approximated with the expression

$$
\beta_{2}(z)=\left\langle\beta_{2}\right\rangle+\beta_{2}^{A} \sin (2 \pi z / \Lambda+\phi)
$$

where $\left\langle\beta_{2}\right\rangle, \quad \beta_{2}^{A}$ and $\Lambda$ are the average normal dispersion of the fiber, the amplitude and the spatial period of the dispersion fluctuations, respectively. We have numerically found that $\phi$, which is a phase shift of the sinusoidal dispersion modulation, does not significantly influence the spectrum at the output of the DOF: therefore we have also neglected this term in our analysis. 
As well known, in the normal dispersion regime a CW pump of power $P$ in a DOF leads to a QPM-FWM sidebands at relative frequencies [4] :

$$
\Omega_{p}= \pm \sqrt{\frac{2 \pi p / \Lambda-2 \gamma P}{\left\langle\beta_{2}\right\rangle}}
$$

where $p= \pm 1, \pm 2, \pm 3 \ldots$. More recently [5] it has been shown that the gain undergone by the $\mathrm{p}^{\text {th }}$ sidebands after a propagation length $L$ can be predicted by the formula

$$
G_{p}^{d B}=10 \log _{10}\left(2 \gamma P L J_{p}\left(\frac{\beta_{2}^{A} \Omega_{p}^{2}}{2 \pi / \Lambda}\right)\right)
$$

where $J_{p}$ is the Bessel function of order $\mathrm{p}$.
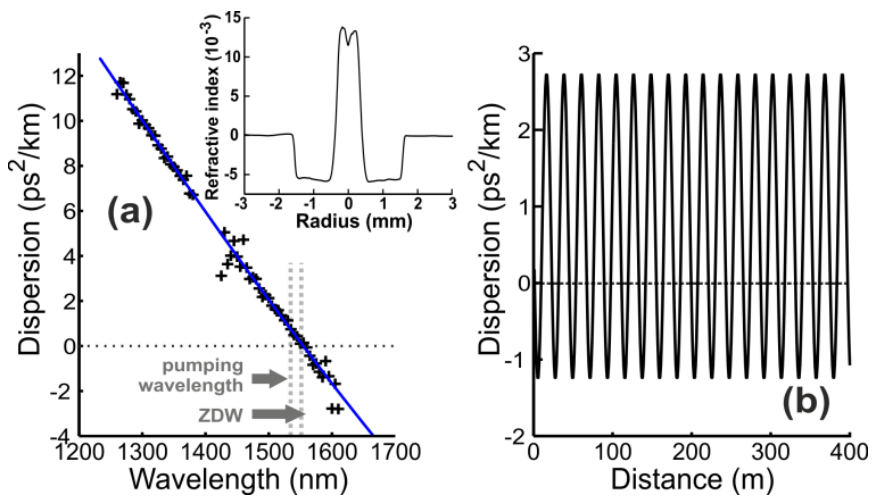

Fig. 1. (a) Measured values of average GVD (crosses) vs. wavelength, with linear approximation as a guide to the eye (solid curve). The refractive index profile of the fiber preform is plotted as an inset. (b) Estimated periodic variation of the GVD at the pump wavelength of $1534.7 \mathrm{~nm}$.

In order to achieve and study the generation of unequal spacing frequency sidebands according to the relation of Eq. (3), we have designed and fabricated a highly nonlinear DOF with a zero dispersion wavelength (ZDW) in the telecom $\mathrm{C}$ band. The fiber preform was prepared by the MCVD process using mixtures of $\mathrm{GeCl}_{4}$, $\mathrm{SiCl}_{4}$ and $\mathrm{SiF}_{4}$ in order to obtain the necessary refractive index profile (see inset of Fig. 1), and it was drawn into a $400 \mathrm{~m}$ long highly nonlinear DOF. Fig. 1(a) shows the measured values of the average dispersion of the fiber as a function of wavelength. As it can be seen, the average fiber GVD remains normal for wavelengths below the ZDW which is located at about $1556 \mathrm{~nm}$. On the other hand, Fig. 1(b) shows the estimated periodic variation with distance of the GVD $\beta_{2}$ at the pump wavelength of $1534.7 \mathrm{~nm}$ : the imposed dispersion oscillation period $\Lambda$ was equal to $20 \mathrm{~m}$, and the resulting GVD oscillation amplitude is estimated as $\beta_{2}^{A}=2 \mathrm{ps}^{2} / \mathrm{km}$, with an average value $\left\langle\beta_{2}\right\rangle$ of $0.74 \mathrm{ps}^{2} / \mathrm{km}$. The nonlinear and loss coefficients are $10 \mathrm{~W}^{-1} \cdot \mathrm{km}^{-1}$ and $0.7 \mathrm{~dB} / \mathrm{km}$, respectively.
In the experiments, we pumped the DOF with a microchip laser emitting $3.7 \mathrm{~ns}$ pulses, with a repetition rate of $2.68 \mathrm{kHz}$. Given the ns duration of the pulses, we can assimilate the pump to a quasi- $\mathrm{CW}$ source having a power close to the peak power of the pulses. As the power was raised above $\mathrm{P}=5 \mathrm{~W}$, a primary set of spectral sidebands was generated involving multiple sidebands with unequal frequency spacing on either side of the pump (see Fig. 2(a)). Moreover, a second set of sidebands having a regular frequency spacing $\Omega_{1}$ is superimposed on the first one. We may associate this secondary set to cascade or MFWM initiated by the mixing between the pump and the first QPM sidebands. We may also note additional sidebands located between the $1^{\text {st }}$ QPM sideband and the pump, that we attribute to FWM between the $1^{\text {st }}$ and the $2^{\text {nd }}$ QPM sidebands. Whenever the pump power increased above $\mathrm{P}=8 \mathrm{~W}$, the frequency peaks merge into a nearly flat $\mathrm{SC}$, and the nonlinear Raman contribution becomes a significant factor.
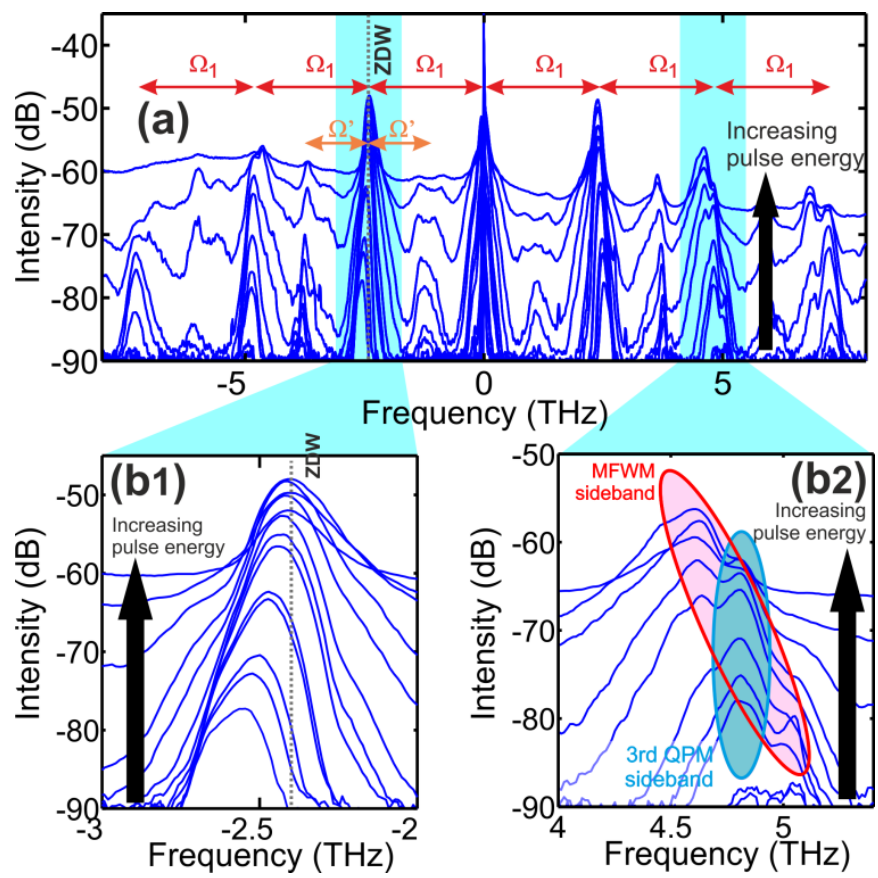

Fig. 2. Sideband intensities vs frequency detuning from the pump, showing: (a) the variation of the output spectrum vs input pump power in the range of $4 \mathrm{~W}-10 \mathrm{~W}$. Magnification of the evolution with pump energy of the first and third sidebands is provided in panels (b1) and (b2), respectively.

Details of the evolution of the first and third sidebands are provided in panels (b1) and (b2) of Fig.2, respectively. These panels qualitatively describe the influence of pump power on both the position and shape of the generated sidebands. Contrary to the well-known MI process occurring in the anomalous dispersion regime of uniform dispersion fibers, increasing here the input pump power leads to sidebands which are closer to the pump. The $1^{\text {st }}$ QPM Stokes sideband in Fig.2(b1) is located in the close vicinity of the fiber ZDW (see the dashed vertical line), which enhances the efficiency of additional competing FWM processes. We may also clearly notice in Fig.2(b1) that the tail of the $1^{\text {st }}$ QPM Stokes sideband undergoes a 
strong and asymmetric spectral broadening when crossing the ZDW. On the other hand, the third QPM sideband in Fig.2(b2) exhibits a double peak structure, where each peak has a different growth rate as the pump power increases. As we shall see, the two sideband peaks in Fig.2(b2) originate from two distinct and competing FWM processes, namely QPM and MFWM.

A more quantitative analysis of the sideband dependence upon pump power is provided in Fig. 3, where the analytical results of Eqs. (3) and (4) are compared with the experimental measurements and the numerical integration of the NLSE (1). From Fig. 3(a) we may point out a good agreement between theory and experiments for the frequency location of both the $1^{\text {st }}$ and $2^{\text {nd }}$ QPM sidebands: the experimental results are well within the frequency range which is predicted by the numerics. The evolution of the third sideband, which exhibits a much larger bandwidth, follows the predictions of Eq. (3) at relatively low pump powers (i.e., for $\mathrm{P}<6 \mathrm{~W}$ ). Whereas at higher pump powers the peak gain frequency shifts towards lower frequencies, in qualitative agreement with the detuning corresponding to the MFWM process resulting from the FWM of the pump and the $1^{\text {st }} \mathrm{QPM}$ sideband, namely $2 \Omega_{1}$.
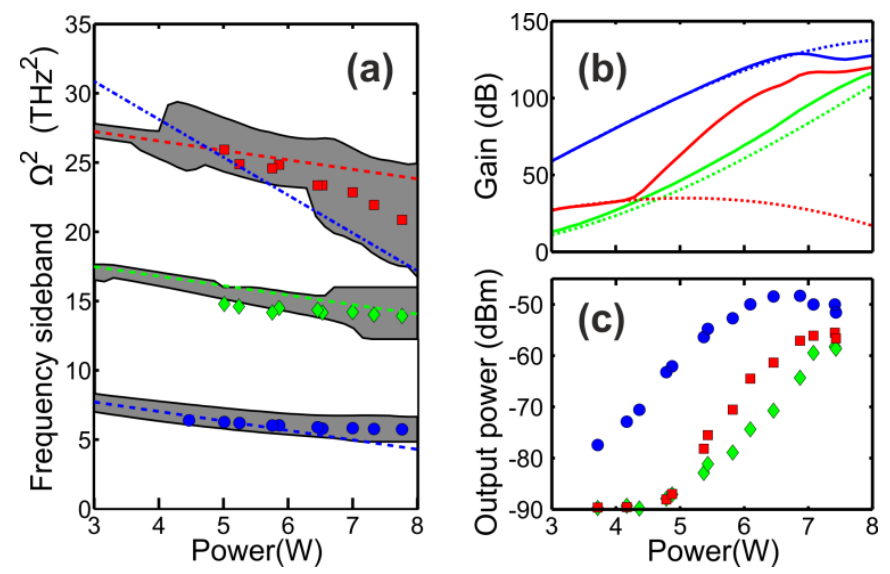

Fig. 3. Comparison of the experimental, numerical and analytical dependence of first three QPM sidebands upon pump power P. Results are plotted in blue, green and red for the $1^{\text {st }}, 2^{\text {nd }}$ and $3^{\text {rd }}$ sideband, respectively. Experimental results are plotted with filled markers, whereas analytical results from Eqs. (3) or (4) are plotted with dotted lines. For the $3^{\text {rd }}$ sideband, the measured data correspond to either the maximum of the $3^{\text {rd }} \mathrm{QPM}$ sideband or of the FWM sideband once they merge. (a) Evolution of the sideband frequencies. Grey areas correspond to the $10 \mathrm{~dB}$ bandwidth of the numerically obtained sidebands. The dashed dotted line represents the frequency resulting from FWM of the pump and the $1^{\text {st }}$ QPM sideband. (b) Comparison of the gain obtained from numerical simulation (solid lines) with the predictions of Eq (4). (c) Experimental pump power dependence of spectral component peak power for the various sidebands.

The dependence of the sidebands gain upon input pump power $\mathrm{P}$ is plotted in Fig. 3(b). Results from numerical simulations (solid lines) are in reasonable agreement with the results predicted by Eq. (4) (dotted lines) for the first and second sidebands. However, we notice that the agreement is limited to low powers only for the third sidebands. Indeed for input pump peak powers above $\mathrm{P}=4 \mathrm{~W}$, instead of experiencing the gain drop which is predicted by Eq. (4), a second process, i.e., the MFWM between the pump and the first sideband, effectively boosts the $3^{\text {rd }}$ sideband gain. The experimental evolution of the spectral component peak power of the first three sidebands qualitatively confirms these numerical trends. Indeed, as shown in Fig. 3(c), the sideband intensity grows rapidly larger with increasing pump power: nearly $20 \mathrm{~dB}$ of sideband gain is obtained for a $1 \mathrm{~dB}$ increase of pump power. Fig. 3(c) also confirms that the $3^{\text {rd }}$ sideband experiences a larger gain than the $2^{\text {nd }}$ sideband, which is not consistent with the prediction of Eq. (4) that does not take into account the presence of MFWM.

In Fig. 4(a) we summarize the experimental measurements carried out with increasing input pump power. The development of the various sidebands originating from either the QPM or the MFWM processes is well reproduced by numerically solving the NLSE (1) (see Fig. 4(b)). This validates the various assumptions that we made for the clarity of the discussion. Among those assumptions, it is worth noting that, in spite of the frequency dependence of the GVD shown in Fig. 1(a), and of the fact that a non-negligible part of the spectrum falls within the region of anomalous dispersion, it appears that neglecting higher order dispersive terms is a fully reasonable approximation. In other words, the consequences on the nonlinear wave dynamics of the longitudinal variation of $\beta_{2}$ are here much more significant than the frequency dependence of $\left\langle\beta_{2}\right\rangle$.
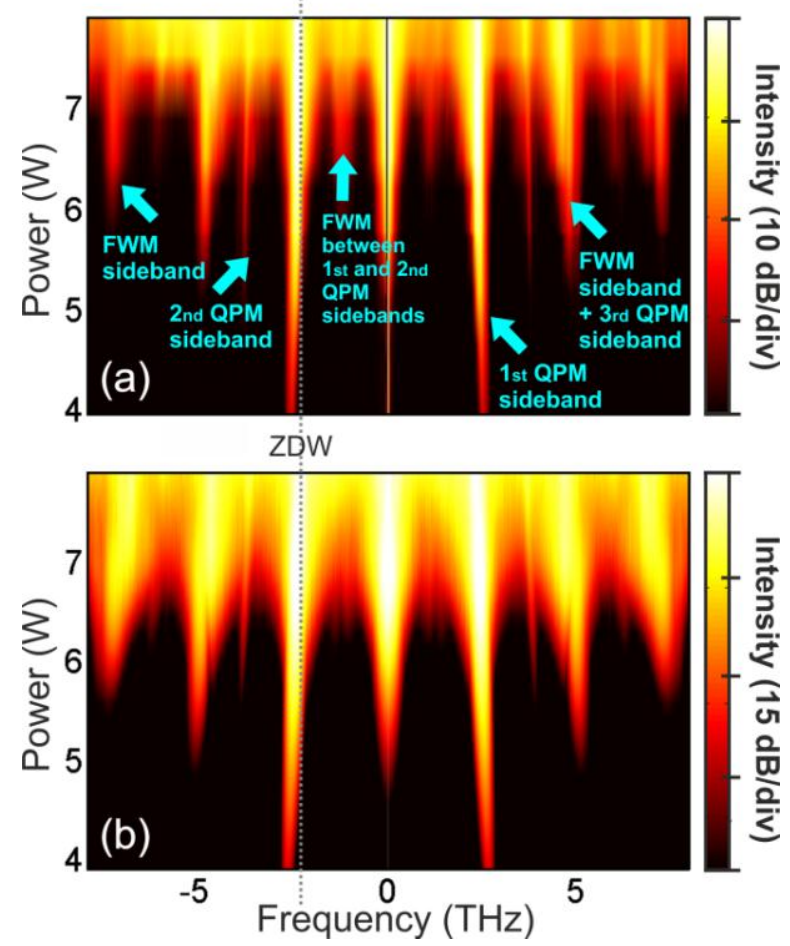

Fig. 4. Contour plot of (a) experimental and (b) numerical spectral intensity vs. input pump power. Spectra obtained from simulations are averaged over 20 shots. 
We have complemented our measurement studies by experimentally investigating the FWM dynamics with partially coherent pumping. Therefore we replaced the nanosecond microchip laser by a home-made source based on the amplification of spectrally filtered continuous amplified spontaneous emission [10]. The spectral full width at half maximum of the optical band pass Gaussian filter centered at $1534.37 \mathrm{~nm}$ was tuned between 10 and $200 \mathrm{GHz}$. The influence of the average power of the partially incoherent field with a $20-\mathrm{GHz}$ spectral bandwidth on the generated FWM spectrum is summarized in Fig. 5(a). When compared with the previously discussed coherent case, one may point out several different features. For average powers as low as 1W, several QPM and MFWM sidebands can already be distinguished. This contrasts with Fig. 4, where peak powers exceeding $5 \mathrm{~W}$ were required in the coherent case in order to observe the cascading of the FWM process. The $2^{\text {nd }}$ QPM-FWM sideband is already clearly apparent as soon as $0.5 \mathrm{~W}$ of average power is reached, whereas Eq. (4) predicts a gain below the $\mathrm{dB}$ level in the case of fully coherent pumping. As far as the frequency of the sidebands is concerned, we may note that for $1 \mathrm{~W}$ of pump power the $1^{\text {st }} \mathrm{QPM}$ sideband is detuned by $2.7 \mathrm{THz}$ from the pump frequency, which is lower than the analytical prediction of Eq. (3), i.e. $3 \mathrm{THz}$.

Moreover, in the partially coherent case the different sidebands are significantly broader than those recorded in the coherent case. Subplot 5(b) reveals the influence of the input source spectral bandwidth on the sideband spectrum. As it can be seen, increasing the source incoherence while maintaining its average power fixed leads to a drop of the spectral gain, as well as to a modification of the spectral sidebands. All of those different features are in qualitative agreement with numerical simulations (not reported here) of the NLSE (1) with incoherent pumping. These results confirm that the source incoherence has a dramatic influence on parametric processes in optical fibers. The strong fluctuations of the instantaneous temporal intensity of the partially coherent pump associated with the high powerdependence of the sideband gain explain in part such a behavior and may also lead the emergence of extreme events. [10]. A more detailed description of the impact of the incoherence is beyond the scope of the present study, and will be the subject of further investigations.

In conclusion, we proposed and demonstrated the use of a ns-pulse pumped DOF for the generation of a comb of multiple spectral sidebands in the telecom $\mathrm{C}$-band. We observed multiple QPM-FWM peaks mixed with standard cascade FWM sidebands. The observation of such competition is made possible by the unequal spacing of QPM-FWM sidebands, in contrast with the equally spaced MFWM sidebands. This example confirms all the potentialities brought by the interplay of the QPM-FWM process with better known parametric interactions [11, 12 . We also experimentally investigated the generation of modulational instability and FMW sidebands under partially coherent pumping. We found that in this case a nearly 6 -fold reduction in the average power needed to reach a detectable level for the QPM sidebands is obtained. In this context, using a partially coherent source such as a fiber Raman laser may become an attractive solution.
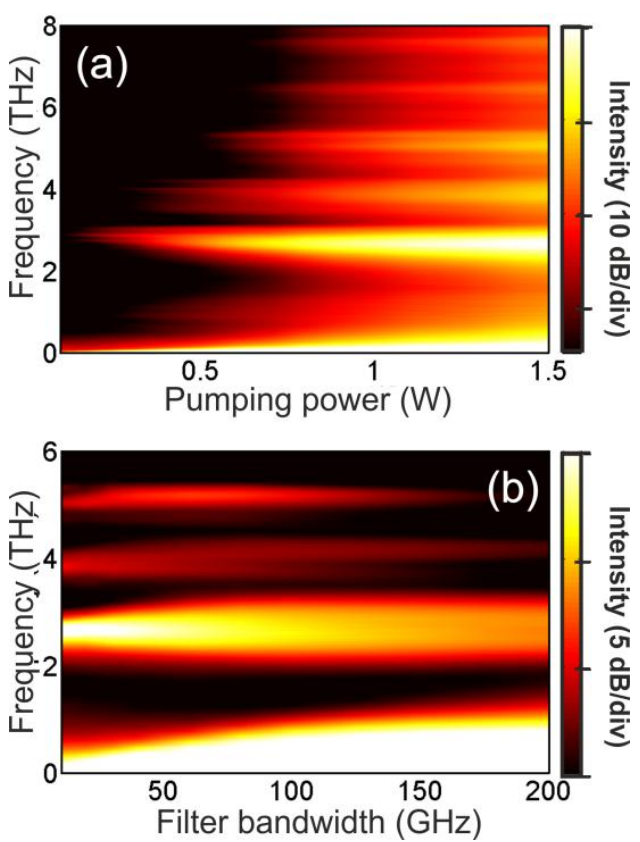

Fig. 5. (a) Experimental variation of the output spectrum vs pump power for an initial incoherence width of $20 \mathrm{GHz}$. (b) Evolution of the output spectrum vs the incoherence width of the pump (as defined by the filter width) for a fixed input average power of $1.5 \mathrm{~W}$.

We acknowledge the financial support of the Conseil Regional de Bourgogne (Pari Photcom) and the funding of the Labex ACTION program (ANR-11-LABX-01-01). The experimental work has benefited from the PICASSO Platform of the University of Burgundy. This research was funded by the European Research Council under Grant Agreement 306633 and by Fondazione Cariplo, grant n.2011-0395. A.S. acknowledges support from a Landau Network-Cariplo Foundation fellowship and from the Ministry of Education and Science of the Russian Federation.

\section{References.}

1. T. J. Kippenberg, R. Holzwarth, and S. Diddams, Science 332, 555 (2011).

2. M. Haelterman, S. Trillo, and S. Wabnitz, Opt. Lett. 17, 745 (1992)

3. A. B. Matsko, A. A. Savchenkov, and L. Maleki, Opt. Lett. 37, 43-45 (2012).

4. N. J. Smith and N. J. Doran, Opt. Lett. 21, 570 (1996).

5. M. Droques, A. Kudlinski, G. Bouwmans, G. Martinelli, and A. Mussot, Phys. Rev. A 87, 013813 (2013).

6. A. Armaroli and F. Biancalana, Opt. Express 20, 2509625110 (2012).

7. F. K. Abdullaev, S. A. Darmanyan, A. Kobyakov, and F. Lederer, Phys. Lett. A 220, 213-218 (1996).

8. M. Droques, A. Kudlinski, G. Bouwmans, G. Martinelli, and A. Mussot, Opt. Lett. 37, 4832-4834 (2012).

9. A. A. Sysoliatin, M. Y. Salganskii, G. Manili, D. Modotto, and S. Wabnitz, in ECOC 2012, 2012), Th.2.E.2. 
10. K. Hammani, C. Finot, and G. Millot, Opt. Lett. 34, 1138-1140 (2009).

11. M. Droques, A. Kudlinski, G. Bouwmans, G. Martinelli, A. Mussot, A. Armaroli, and F. Biancalana, Opt. Lett. 38, 3464-3467 (2013).

12. A. Armaroli and F. Biancalana, Phys. Rev. A 87, 063848 (2013). 


\section{References with title.}

1. T. J. Kippenberg, R. Holzwarth, and S. Diddams, "Microresonator-based optical frequency combs," Science 332, 555 (2011).

2. M. Haelterman, S. Trillo, and S. Wabnitz, "Additive-modulation-instability ring laser in the normal dispersion regime of a fiber," Opt. Lett. 17, 745 (1992).

3. A. B. Matsko, A. A. Savchenkov, and L. Maleki, "Normal group-velocity dispersion Kerr frequency comb," Opt. Lett. 37, 43-45 (2012).

4. N. J. Smith and N. J. Doran, "Modulational instabilities in fibers with periodic dispersion management," Opt. Lett. 21, 570 (1996).

5. M. Droques, A. Kudlinski, G. Bouwmans, G. Martinelli, and A. Mussot, "Dynamics of the modulation instability spectrum in optical fibers with oscillating dispersion," Phys. Rev. A 87, 013813 (2013).

6. A. Armaroli and F. Biancalana, "Tunable modulational instability sidebands via parametric resonance in periodically tapered optical fibers," Opt. Express 20, 25096-25110 (2012).

7. F. K. Abdullaev, S. A. Darmanyan, A. Kobyakov, and F. Lederer, "Modulational instability in optical fibers with variable dispersion," Phys. Lett. A 220, 213-218 (1996).

8. M. Droques, A. Kudlinski, G. Bouwmans, G. Martinelli, and A. Mussot, "Experimental demonstration of modulation instability in an optical fiber with a periodic dispersion landscape," Opt. Lett. 37, 4832-4834 (2012).

9. A. A. Sysoliatin, M. Y. Salganskii, G. Manili, D. Modotto, and S. Wabnitz, "Supercontinuum frequency comb from dispersion oscillating optical fiber," in ECOC 2012, 2012), Th.2.E.2.

10. K. Hammani, C. Finot, and G. Millot, "Emergence of extreme events in fiber-based parametric processes driven by a partially incoherent wave," Opt. Lett. 34, 1138-1140 (2009).

11. M. Droques, A. Kudlinski, G. Bouwmans, G. Martinelli, A. Mussot, A. Armaroli, and F. Biancalana, "Fourth-order dispersion mediated modulation instability in dispersion oscillating fibers," Opt. Lett. 38, 3464-3467 (2013).

12. A. Armaroli and F. Biancalana, "Vector modulational instability induced by parametric resonance in periodically tapered highly- 